\title{
REPRODUCIBILITY OF CUTANEOUS VASCULAR CONDUCTANCE RESPONSES TO SLOW LOCAL HEATING ASSESSED USING 7-LASER ARRAY PROBES
}

\author{
Ellen A. Dawson ${ }^{1}$ \\ David A. Low ${ }^{1}$ \\ Iris H.M. Meeuwis ${ }^{1}$ \\ Floor G. Kerstens ${ }^{1}$ \\ Ceri L. Atkinson ${ }^{3}$ \\ N. Timothy Cable ${ }^{1}$ \\ Daniel J. Green ${ }^{1,3}$ \\ Dick H.J. Thijssen ${ }^{1,2}$
}

${ }^{1}$ Research Institute for Sport and Exercise Science, Liverpool John Moores University, Tom Reilly Building, Byrom Street, Liverpool L3 3AF, United Kingdom

${ }^{2}$ Department of Physiology,

Radboud University Nijmegen Medical Centre, the Netherlands

${ }^{3}$ School of Sport Science, Exercise and Health,

The University of Western Australia, Crawley, Western Australia, 6009

\author{
Running Title: \\ Reproducibility of skin blood flow
}

Total number of words: $\mathbf{5 3 5 0}$

Total number of figures: 2

Total number of tables: 4

\section{Author for Correspondence:}

Dick HJ Thijssen

Professor of Cardiovascular Physiology and Exercise

Research Institute for Sport and Exercise Sciences

Liverpool John Moores University, Byrom Street, L3 3AF, Liverpool (United Kingdom)

Email: d.thijssen@ljmu.ac.uk 


\section{ABSTRACT}

Objective. Gradual local heating of the skin induces a largely nitric oxide(NO)-mediated vasodilatation. However, use of this assessment of microvascular health is limited because little is known about its reproducibility.

Methods. Healthy volunteers ( $n=9)$ reported twice to the laboratory. Cutaneous vascular conductance (CVC), derived from laser Doppler flux and mean arterial pressure, was examined in response to a standardised local heating protocol $\left(0.5^{\circ} \mathrm{C}\right.$ per 150 s from $33-42^{\circ} \mathrm{C}$, followed by 20 -minutes at $44^{\circ} \mathrm{C}$ ). Skin responses were examined at two locations on the forearm (betweensite). Heating was repeated after a break of 24-72 hours (between-day). Reproducibility of skinresponses at $33-42^{\circ} \mathrm{C}$ is presented for absolute $\mathrm{CVC}$ and relative CVC-responses corrected for maximal $\mathrm{CVC}$ at $44^{\circ} \mathrm{C}\left(\% \mathrm{CVC}_{\max }\right)$.

Results. Between-day reproducibility of baseline $\mathrm{CVC}$ and $\% \mathrm{CVC}_{\max }$ for both sites was relatively poor (22-30\%). At $42^{\circ} \mathrm{C}, \mathrm{CVC}$ and $\% \mathrm{CVC}_{\max }$ responses showed less variation (9-19\%), whilst absolute CVC-responses at $44^{\circ} \mathrm{C}$ were $14-17 \%$. Between-day variation for $\% \mathrm{CVCmax}$ increased when using data from site 1 on day 1 , but site 2 on the subsequent day $(25 \%)$.

Conclusion. Day-to-day reproducibility of baseline laser Doppler-derived skin perfusion responses is poor, but acceptable when absolute and relative skin perfusion to a local gradual heating protocol is utilised and site-to-site variation is minimised.

Keywords: skin microcirculation; endothelial function; nitric oxide; local heating; microvasculature

\section{ABBREVIATIONS}




$\begin{array}{ll}\text { AR } & \text { Axon-reflex } \\ \text { CV } & \text { Coefficient of variation } \\ \text { CVC } & \text { Cutaneous Vascular Conductance } \\ \text { \%CVCmax } & \text { CVC normalised to the maximal flux achieved during } 44^{\circ} \mathrm{C} \\ \text { LDF } & \text { Laser Doppler Flowmetry } \\ \text { NO } & \text { Nitric Oxide } \\ \text { PU } & \text { Perfusion units } \\ \text { RBCF } & \text { Red blood cell flux }\end{array}$




\section{INTRODUCTION}

Recent studies suggest that microvascular abnormalities may represent the detectable manifestation of future cardiovascular disease, preceding abnormalities in larger conduit arteries and arterioles $[2,4,18,23,29]$. These observations support the clinical relevance of examining microvascular function. The cutaneous microcirculation is an easily accessible microvascular bed which is believed to reflect systemic microcirculatory function and health. Using laserDoppler flowmetry (LDF), previous studies have examined skin responses to local heating protocols to explore (skin) microcirculatory function.

The cutaneous microcirculation in humans is under direct influence of neurogenic reflexes (e.g. axon-reflex) and locally released substances (e.g. nitric oxide (NO)), which contribute to the vasodilation that occurs during local heating $[3,8,10,11,20]$. The NO-mediated dilation is of special importance as NO is a potent anti-atherogenic agent, and inability of the endothelium to produce NO is an early atherogenic event [3,4,29]. Crucially, the rate of skin heating seems to alter the contribution of NO to local heating responses. Rapidly heating the skin by $0.5^{\circ} \mathrm{C}$ per 5 seconds causes an axon-reflex (AR)-mediated vasodilation, followed by gradual vasodilation that is perhaps 60-70\% NO-mediated [3,17,20]. More recently, Black et al. found that slowly heating the skin (i.e. $0.5^{\circ} \mathrm{C} / 5$-min) avoids $\mathrm{AR}$ evocation and results in a largely NO-mediated vasodilatation [3]. Whilst the reproducibility of rapid heating protocols has been demonstrated to reach acceptable levels [12,25], no previous study explored the reproducibility of the largely NO-dependent, slow heating protocol introduced by Black and colleagues [3]. Such information is of particular importance in repeated measures experiments which aim to as the effect of interventions on microvascular function and health. We therefore examined the between-day 
variability of cutaneous vascular conductance measures derived in response to gradual skin heating in healthy subjects.

LDF is typically performed across a very small portion of the skin, which makes it difficult to use anatomical landmarks to ensure similar placement when performing repeated measurements. A significant portion of the between-day variation of cutaneous vascular conductance (CVC) responses to local heating may relate to errors in site re-selection. Indeed, significant betweensite variation is described for baseline CVC [7,14,27]. Since relatively little is known about the importance of variation in location on the CVC responses to local heating, we also assessed the between-site variation of assessment of cutaneous flow responses to local heating.

\section{MATERIALS AND METHODS}

\section{Subjects}

Nine (male N=5, female N=4) healthy subjects (aged 18-40 years) were recruited. Subjects with a history of cardiovascular disease, hypertension (systolic blood pressure $\geq 140 \mathrm{mmHg}$, diastolic blood pressure $\geq 90 \mathrm{mmHg}$ [1] or hypercholesterolemia (total cholesterol $>6.5 \mathrm{mmol} / \mathrm{l}[22]$, as well as regular smokers, were excluded. We also excluded individuals on drugs that influence the cardiovascular system and individuals taking vitamin or any other supplements. All females were tested during the early follicular phase of their menstrual cycle, because of the known hormonal influences on skin blood flow [5]. All procedures undertaken within this investigation were approved by the Ethics Committee of Liverpool John Moores University. This study was carried 
out in accordance with the Declaration of Helsinki. Written informed consent was obtained from all subjects.

\section{Experimental Design}

All subjects reported twice to our laboratory. All studies were conducted in a quiet, temperature controlled environment. Subjects arrived after fasting for at least 6 hours and they were instructed to abstain from alcohol, caffeine and exercise for $24 \mathrm{~h}$ prior to testing. Upon arrival, subjects were rested in the supine position on a bed and were instrumented for LDF measurements. Following a 20-minute rest period, baseline heart rate and blood pressure were assessed using an automated sphygmomanometer. Forearm skin responses to local heating $\left(0.5^{\circ} \mathrm{C} / 150\right.$ s from $33^{\circ} \mathrm{C}$ to $42^{\circ} \mathrm{C}$, followed by a 20 -minute period at $\left.44^{\circ} \mathrm{C}\right)$ were examined simultaneously at two distinct locations on the dominant forearm, separated by $\sim 5 \mathrm{~cm}$, to allow for the assessment of between-site variation. After the protocol, the location of both assessment sites was marked, digital photos taken, and measurement of distances from anatomical landmarks recorded to ensure similar re-selection of the probe placement on the subsequent day. On Day 2, separated by at least 24-h (and a maximum of 72 hours) from Day 1, we repeated assessment of the CVC-responses to local heating at both locations. For each subject, all sessions were conducted at the same time of the day to eliminate the possible effects of the circadian rhythm on vascular function [15].

\section{Experimental Measures}

Laser Doppler Flowmetry and local heating protocol. Two sites on the volar aspect of the dominant forearm were randomly chosen (avoiding visible veins) and separated by $\sim 5 \mathrm{~cm}$. If 
necessary, laser Doppler probe sites on the forearm were shaved and cleaned $24 \mathrm{~h}$ prior to testing to avoid any inflammatory response that would otherwise affect skin blood flow. At these sites, cutaneous blood flow was measured as cutaneous red blood cell flux (RBCF) using a laser Doppler flowmeter (Periflux system 5000, Perimed AB, Järfälla-Stockholm, Sweden) and LDF 7-laser array probes (model PF 413, Perimed AB). Local thermal hyperaemia was induced placing a heater disc on the selected forearm sites (Perimed 455, Perimed AB) which were connected to a heating unit (Peritemp 4005 heater, Perimed AB). Calibration of the laser Doppler probes was undertaken before and after the experiments using two generic points, 0 and 250 PU, in accordance with calibration guidelines using a zeroing disk and motility standard (Perimed AB, Sweden). Laser Doppler probes were placed into the heater disc and both heaters and probes were firmly attached to the skin using double-sided stickers and medical tape.

Baseline skin blood flow measures were recorded with the local heating disc temperatures set at $33^{\circ} \mathrm{C}$ for 10 minutes. The temperature was then slowly raised in steps of $0.5^{\circ} \mathrm{C}$ per 150 s to $42^{\circ} \mathrm{C}$ [3]. This was followed by a continuous recording at $42^{\circ} \mathrm{C}$ for $30 \mathrm{~min}$. Subsequently, after confirming the presence of a perfusion plateau, temperature was increased to $44^{\circ} \mathrm{C}$ for an additional 20 minutes to obtain an index of maximal cutaneous blood flow $[13,16,30]$.

Hemodynamics. Heart rate and blood pressure were recorded at the beginning and at the end of the 20-minute resting period using the arm distinct from that used for LDF measurements. Subsequently, mean arterial pressure and heart rate were recorded every 5 minutes during the local heating protocol using a Dinamap automatic monitor (Dinamap V100, GE Healthcare, UK). These repeated blood pressure measurements were used to calculate cutaneous vascular 
conductance (CVC) for the relevant subsequent 5 minute period, which accounts for skin blood flow changes which occur as a result of changes in blood pressure [7,20].

\section{Data analysis}

Cutaneous red blood cell flux is expressed in perfusion units (PUs) and reflects local cutaneous blood flow. The change in cutaneous blood flow is expressed as cutaneous vascular conductance (CVC), defined as flux divided by mean arterial pressure (in PU/mmHg) [7]. Baseline laser Doppler flux was averaged over 10 minutes. The plateau phases during heating at $42^{\circ} \mathrm{C}$ and $44^{\circ} \mathrm{C}$ were averaged over the last 5-minutes of the protocol. Positive and negative spikes in the data points, due to unwanted movement of the subject, were identified and deleted. Subsequently, data were presented as absolute flux $\left(\mathrm{CVC}\right.$; baseline, $42^{\circ} \mathrm{C}$ and $\left.44^{\circ} \mathrm{C}\right)$, whilst data at baseline and $42^{\circ} \mathrm{C}$ were also normalised to the maximum flux achieved during $44^{\circ} \mathrm{C}\left(\% \mathrm{CVC}_{\max }\right)$. All data were collected in LabChart 7.0 (ADInstruments, Dunedin, New Zealand).

\section{Statistics}

Data were expressed as mean \pm SD. Statistical significance was set at $\mathrm{P}<0.05$. The coefficient of variation $(\mathrm{CV})$ was calculated to test the between-day reproducibility of the $\mathrm{CVC}$ and $\% \mathrm{CVC}_{\max }$ at baseline, $42^{\circ} \mathrm{C}$ and $44^{\circ} \mathrm{C}$ for both sites $[11,25,31]$. To examine between-site variation, $\mathrm{CV}$ was calculated by comparing site 1 versus site 2 at the different time-points (baseline, $42^{\circ} \mathrm{C}$ and $44^{\circ} \mathrm{C}$ ), and by calculating the between-day reproducibility using site 1 and site 2 .

Before calculating the $\mathrm{CV}$, a natural logarithmic transformation was applied to correct for heteroscedasticity of the data. The $\mathrm{CV}$ was calculated following the classical approach based on 
the pooled SD. Briefly, the root mean square of the error term (square root of the error term of the adjusted mean squares) of an ANOVA was used to calculate the pooled variance. After logtransformation, the $\mathrm{CV}$ was calculated by $\sqrt{ }[\exp (\mathrm{var})-1] \times 100$, where $\exp (\mathrm{var})$ is the exponent of the pooled variation. In addition, a 95\% CI (confidence interval) of the CV was calculated, based on the variation of the log-transformed data and the $\chi^{2}$ distribution $(\sqrt{[}[$ exponent $($ degrees of freedom $\times$ variation $/ \chi 2$ distribution) -1$] \times 100$ ). A CV of $<10 \%$ is considered good and $<20 \%$ is acceptable for biological variables [28]. Bland Altman plots were constructed to demonstrate variability at an individual level. A paired t-test was used to determine significant differences between measures and a linear regression was used to assess proportional bias. A two way ANOVA was used to determine changes in blood pressure. Data were stored and transformed within Microsoft Excel (Microsoft Office 2010, Microsoft Corporation) and SPSS was used to construct Bland-Altman plots and to assess changes in blood pressure (SPSS 22, Chicago, Illinois).

\section{RESULTS}

\section{Study population}

Participant characteristics are presented in Table 1. Figure $\mathbf{1}$ shows a representative skin blood flow trace during the local heating protocol with the 3 times periods (at 33,42 and $44{ }^{\circ} \mathrm{C}$ ) that were used for data analysis clearly indicated. There was no significant change in mean arterial blood pressure throughout the heating protocol $(\mathrm{P}=0.41)$ and no difference between days $(\mathrm{P}=0.53)$ and no interaction $(\mathrm{P}=0.36)$. 


\section{Between-day reproducibility}

Baseline. Average baseline skin perfusion across both sites and days varied between $18 \pm 7$ to $31 \pm 11$ when presented as perfusion units (PU) and $7 \pm 2$ to $10 \pm 4$ when corrected for maximal perfusion (in \% $\mathrm{PU}_{\max }$ ). Baseline $\mathrm{CVC}$ across both sites and days ranged from $0.23 \pm 0.09$ to $0.40 \pm 0.18$ (in arbitrary units $\mathrm{AU} / \mathrm{mmHg}$ ) and from $7 \pm 2$ to $11 \pm 4$ when corrected to maximal perfusion (in $\% \mathrm{CVC}_{\max }$ ). Between-day variation of baseline perfusion/CVC ranged between 19.6-30.9\%, with no consistent differences in $\mathrm{CV}$ when presented as absolute or relative values, or when presented in PU or CVC units (Table 2).

Local heating to 42 and $44^{\circ} \mathrm{C}$. Between-day reproducibility of forearm skin perfusion expressed as absolute PU at $42^{\circ} \mathrm{C}$ showed acceptable variation for both sites (18.1 and $16.9 \%$, Table 3). Reproducibility was further improved when data were presented after correcting for maximal skin perfusion (8.4 and 10.3\%, for sites 1 and 2 respectively, Table 3 ). Comparable findings were found when data were presented as CVC (Table 3). Bland-Altman plots demonstrate no obvious heteroscedasticity (Figure 2). Between-day reproducibility of the maximal heating response to $44^{\circ} \mathrm{C}$ (for PU and CVC) was acceptable and ranged from 14.7 and $18.1 \%$ (Table 3).

\section{Between-site reproducibility}

Baseline. Poor agreement was evident between sites 1 and 2 when examining baseline forearm flux or baseline CVC skin perfusion (Table 4). CVs ranged between 29.9 and 30.9\% when examining the between-day reproducibility when using site 1 on day 1 and site 2 on day 2 (Table 4). 
Local heating to 42 and $44^{\circ} \mathrm{C}$. The CVs between site 1 and 2 showed good agreement during the $42^{\circ} \mathrm{C}$-plateau phase when data were corrected for the maximal heating response $(\% \mathrm{CVC}$ max, $6.3 \%$ on day 1 and $\mathbf{5 . 0} \%$ on day 2, Table 4). However, CVs between sites were significantly higher when absolute flux or CVC was used (21.3-20.6 for both days; Table 4). When using site 1 on day 1 and site 2 on day 2 as data points, between-day reproducibility was poor for absolute data, but moderate-to-acceptable for relative flux and relative CVC (Table 4).

Comparing both sites during the plateau phase at $44^{\circ} \mathrm{C}, \mathrm{CV}$ for absolute flux and $\mathrm{CVC}$ was $20.3 \%$ and $18.5 \%$ at day 1 and 2, respectively (Table 4). The CV for absolute CVC at $44^{\circ} \mathrm{C}$ was $\mathbf{2 5} \%$ for the between-day variation when using site 1 on day 1 and site 2 on day 2 (Table 4 ).

\section{DISCUSSION}

The aims of this study were to investigate the between-day and between-site reproducibility of NO-mediated skin blood flow responses to a localised, gradual skin heating protocol. We found that baseline laser-Doppler derived cutaneous perfusion measures were variable, with poor between-day reproducibility. Moderate reproducibility was observed for laser-Doppler flux responses to local heating. Importantly, we consistently found that reproducibility improves when presented as a percentage of maximal perfusion (to $44^{\circ} \mathrm{C}$ ). Finally, whilst site specific variability was moderate-to-good, we observed poor site-to-site variation, especially when data were expressed in absolute terms. Although variability was improved when data were corrected for maximal values at $44{ }^{\circ} \mathrm{C}$, our findings largely support to carefully control similar placement of the laser-Doppler probes when performing repeated measurements. This information improves 
our insight into the use of skin perfusion responses to reflect microvascular function and health in humans.

Rapid heating of the skin, an approach introduced by Minson and colleagues [20], demonstrates good-to-moderate perfusion response reproducibility when presented as a percentage of the maximal perfusion (CVs ranged between 4-19\%) [25,31]. In agreement with these studies, we observed that skin responses to gradual heating the skin resulted in good-to-moderate between day reproducibility. Although absolute forearm CVC showed acceptable reproducibility during the plateau phase at $42^{\circ} \mathrm{C}$ and $44^{\circ} \mathrm{C}$, variability of the data at $42^{\circ} \mathrm{C}$ further improved when corrected for maximal $\mathrm{CVC}$ at $44^{\circ} \mathrm{C}$. This indicates that absolute and relative $\mathrm{CVC}$ data during the slow local heating protocol can be used as reliable repeated measures index of skin perfusion. This somewhat contrasts previous work that adopted rapid heating, as they only observed acceptable reproducibility when data was expressed as a percentage of maximal CVC. This difference may relate to the mechanisms by which the slow and rapid heating protocols induce responses. Whilst slowly heating the skin prevents significant axon-reflexes and leads to a largely NO-mediated response, rapidly heating the skin activates the axon-reflex and leads to a NO- and EDHF-mediated response [6,20]. The involvement of multiple vasoactive substances in the rapid heating response may explain the larger variation in absolute perfusion observed in previous studies compared to relatively modest variation of the slow heating protocol observed in our study.

Another aim of our study was to provide better insight into the impact of between-site variability. When measuring skin blood flow with LDF, only a very small portion of the skin is covered by 
the heating disc $\left(\sim 7 \mathrm{~mm}^{2}\right)$ which could affect the site-to-site reliability of the technique. Between-site reproducibility of PU and CVC was poor for baseline perfusion, even after correction for the maximal perfusion. However, a good-to-moderate variability between both sites was found for the plateau phases. This suggests that the spatial resolution of LDF has a larger impact on baseline perfusion compared to vasodilator responses during local heating, especially when data are normalised to maximal perfusion. Furthermore, we also examined whether the reproducibility of the CVC and \%CVCmax changed when using the different sites to assess between-day variation, instead of using the same site. Whilst reproducibility of absolute CVC data was poor, we found acceptable reproducibility when examining levels of \%CVCmax at the $42^{\circ} \mathrm{C}$ plateau phase when using different sites for the between-day variation. Nonetheless, all $\mathrm{CV}$ values were above that observed for the between-day comparison when using the same site. These data highlight the importance of minimising variation in the measurement site but also suggest that, despite using a different measurement sites, analysis of $\% \mathrm{CVCmax}$ at the $42^{\circ} \mathrm{C}$ plateau phase is robust. Nonetheless, the higher $\mathrm{CV}$ with different sites suggests that care should be taken to utilise the same site during repeat measurements. Alternatively, other techniques are available which may help to further limit this variability including laser speckle contrast imaging $[19,24]$.

Limitations. A potential limitation of our study is that we have included a group of healthy volunteers, limiting the extrapolation to groups with cardiovascular risk such as older humans. Although groups with cardiovascular risk or disease typically demonstrate attenuated skin responses to local heating $[3,7,9,21,32]$, a previous study reported similarly good reproducibility of local heating in young and older subjects [31]. Another limitation is that we included men and 
women. Previous studies demonstrated that skin vascular reactivity to local heating is influenced by the menstrual cycle because the increase in progesterone during the luteal phase resets the threshold for active vasodilation in the skin $[5,17]$. To prevent such impact, we examined female subjects $(n=4)$ in their early follicular phase to attenuate the impact of hormonal influences. Furthermore, we allowed for a minimum of 24-h and a maximal interval of 48-h between both testing days in women (and a maximal interval of 72-h in men). Since reproducibility of the data did not markedly differ between men and women, we believe that inclusion of both sexes did not alter the conclusions of our study. Whilst the normalisation to a maximal flow is commonly adopted, some (clinical) populations demonstrate impaired dilator responses to local heating [26]. Consequently, normalisation to the heating response to $44^{\circ} \mathrm{C}$ may not always represent normalisation to the maximal perfusion. Finally, we did not have measurements of skin temperature to ensure that heating was effectively delivered to the skin. However, the characteristic responses suggest that local heating was successful in our subjects.

In conclusion, this is the first study examining the between-day and between-site reproducibility of LDF measurements of the forearm skin during a slow, gradual local heating protocol. We found that assessment of baseline forearm skin perfusion resulted in poor reproducibility, whilst a moderate reproducibility was observed for absolute forearm skin blood perfusion responses to local heating. Reproducibility improved when data were corrected for the maximal perfusion and when site-to-site variation was minimised. These data strongly support the validity of repeated measures of skin perfusion in response to gradual heating protocols in (clinical) studies as a surrogate for microvascular function and health in humans. 


\section{PERSPECTIVES.}

The aim of the study was to assess the reproducibility of a commonly used assessment of nitric oxide-mediated microvascular function in humans. Good reproducibility was reported when skin perfusion responses to localised heating were corrected for maximal perfusion and variation in measurement site was minimised. These data support the use of this technique for both crosssectional and longitudinal studies assessing (patho)physiological adaptation in the human microcirculation. 


\section{AUTHOR CONTRIBUTIONS}

E.A.D, D.A.L., D.J.G. and D.H.J.T conceived and designed the experiment. I.M., F.K., E.A.D., D.A.L., and D.H.J.T. contributed to the collection and analysis of the data along with the writing of the manuscript. All authors helped draft and/or revise the manuscript critically and have approved the final submitted version.

\section{ACKNOWLEDGMENTS}

Dr Thijssen is recipient of the E. Dekker stipend (Netherlands Heart Foundation, 2009 T064)

Prof. Green is funded by the Australian Research Council (DP 130103793).

\section{DISCLOSURES}

None of the authors have conflict of disclosures 


\section{REFERENCES:}

1. Hypertension : quick reference guide : clinical management of primary hypertension in adults. edn. London: National Institute for Health and Clinical Excellence, 2011.

2. Antonios TF, Singer DR, Markandu ND, Mortimer PS, MacGregor GA. Rarefaction of skin capillaries in borderline essential hypertension suggests an early structural abnormality. Hypertension 34: 655-658, 1999.

3. Black MA, Green DJ, Cable NT. Exercise prevents age-related decline in nitric-oxidemediated vasodilator function in cutaneous microvessels. J Physiol 586: 3511-3524, 2008.

4. Bonetti PO, Lerman LO, Lerman A. Endothelial dysfunction: a marker of atherosclerotic risk. Arterioscler Thromb Vasc Biol 23: 168-175, 2003.

5. Charkoudian N, Johnson JM. Female reproductive hormones and thermoregulatory control of skin blood flow. Exerc Sport Sci Rev 28: 108-112, 2000.

6. Choi PJ, Brunt VE, Fujii N, Minson CT. New approach to measure cutaneous microvascular function: an improved test of NO-mediated vasodilation by thermal hyperemia. J Appl Physiol (1985) 117: 277-283, 2014.

7. Cracowski JL, Minson CT, Salvat-Melis M, Halliwill JR. Methodological issues in the assessment of skin microvascular endothelial function in humans. Trends in pharmacological sciences 27: 503-508, 2006.

8. Green DJ, Carter HH, Fitzsimons MG, Cable NT, Thijssen DH, Naylor LH. Obligatory role of hyperaemia and shear stress in microvascular adaptation to repeated heating in humans. J Physiol 588: 1571-1577, 2010.

9. Holowatz LA, Thompson-Torgerson CS, Kenney WL. Altered mechanisms of vasodilation in aged human skin. Exerc Sport Sci Rev 35: 119-125, 2007.

10. Houghton BL, Meendering JR, Wong BJ, Minson CT. Nitric oxide and noradrenaline contribute to the temperature threshold of the axon reflex response to gradual local heating in human skin. J Physiol 572: 811-820, 2006.

11. Huang CS, Wang SF, Tsai YF. Axon reflex-related hyperemia induced by short local heating is reproducible. Microvascular research 84: 351-355, 2012. 
12. Huang CS, Wang SF, Tsai YF. Increasing acclimation period improves the reproducibility of short-heating local thermal hyperemia. Microvascular research 85: 93-98, 2013.

13. Johnson JM, O'Leary DS, Taylor WF, Kosiba W. Effect of local warming on forearm reactive hyperaemia. Clinical physiology 6: 337-346, 1986.

14. Johnson JM, Taylor WF, Shepherd AP, Park MK. Laser-Doppler measurement of skin blood flow: comparison with plethysmography. Journal of applied physiology: respiratory, environmental and exercise physiology 56: 798-803, 1984.

15. Jones H, Green DJ, George K, Atkinson G. Intermittent exercise abolishes the diurnal variation in endothelial-dependent flow-mediated dilation in humans. Am J Physiol Regul Integr Comp Physiol 298: R427-432, 2010.

16. Kellogg DL, Jr., Liu Y, Kosiba IF, O'Donnell D. Role of nitric oxide in the vascular effects of local warming of the skin in humans. J Appl Physiol (1985) 86: 1185-1190, 1999.

17. Lenasi H. Assessment of human skin microcirculation and its endothelial function using laser Doppler flowmetry. In: Medical Imaging, edn, edited by Erondu DOF. Croatia: InTech, 2011, p. 271-296.

18. Levy BI, Ambrosio G, Pries AR, Struijker-Boudier HA. Microcirculation in hypertension: a new target for treatment? Circulation 104: 735-740, 2001.

19. Millet C, Roustit M, Blaise S, Cracowski JL. Comparison between laser speckle contrast imaging and laser Doppler imaging to assess skin blood flow in humans. Microvascular research 82: 147-151, 2011.

20. Minson CT, Berry LT, Joyner MJ. Nitric oxide and neurally mediated regulation of skin blood flow during local heating. J Appl Physiol 91: 1619-1626, 2001.

21. Minson CT, Holowatz LA, Wong BJ, Kenney WL, Wilkins BW. Decreased nitric oxide- and axon reflex-mediated cutaneous vasodilation with age during local heating. J Appl Physiol (1985) 93: 1644-1649, 2002.

22. Reiner Z, Catapano AL, De Backer G, Graham I, Taskinen MR, Wiklund O, Agewall S, Alegria E, Chapman MJ, Durrington P, Erdine S, Halcox J, Hobbs RH, Kjekshus JK, Perrone Filardi P, Riccardi G, Storey RF, David W, Clinical Practice Guidelines Committee of the 
Spanish Society of C. [ESC/EAS Guidelines for the management of dyslipidaemias]. Revista espanola de cardiologia 64: 1168 e1161-1168 e1160, 2011.

23. RG IJ, de Jongh RT, Beijk MA, van Weissenbruch MM, Delemarre-van de Waal HA, Serne $\mathrm{EH}$, Stehouwer CD. Individuals at increased coronary heart disease risk are characterized by an impaired microvascular function in skin. European journal of clinical investigation 33: 536-542, 2003.

24. Rousseau P, Mahe G, Haj-Yassin F, Durand S, Humeau A, Leftheriotis G, Abraham P. Increasing the "region of interest" and "time of interest", both reduce the variability of blood flow measurements using laser speckle contrast imaging. Microvascular research 82: 88-91, 2011.

25. Roustit M, Blaise S, Millet C, Cracowski JL. Reproducibility and methodological issues of skin post-occlusive and thermal hyperemia assessed by single-point laser Doppler flowmetry. Microvascular research 79: 102-108, 2010.

26. Roustit M, Simmons GH, Carpentier P, Cracowski JL. Abnormal digital neurovascular response to local heating in systemic sclerosis. Rheumatology 47: 860-864, 2008.

27. Saumet JL, Kellogg DL, Jr., Taylor WF, Johnson JM. Cutaneous laser-Doppler flowmetry: influence of underlying muscle blood flow. J Appl Physiol (1985) 65: 478-481, 1988.

28. Scott MJ, Randolph PH, Leier CV. Reproducibility of systolic and diastolic time intervals in normal humans: an important issue in clinical cardiovascular pharmacology. J Cardiovasc Pharmacol 13: 125-130, 1989.

29. Sena CM, Pereira AM, Seica R. Endothelial dysfunction - a major mediator of diabetic vascular disease. Biochimica et biophysica acta 1832: 2216-2231, 2013.

30. Taylor WF, Johnson JM, O'Leary D, Park MK. Effect of high local temperature on reflex cutaneous vasodilation. Journal of applied physiology: respiratory, environmental and exercise physiology 57: 191-196, 1984.

31. Tew GA, Klonizakis M, Moss J, Ruddock AD, Saxton JM, Hodges GJ. Reproducibility of cutaneous thermal hyperaemia assessed by laser Doppler flowmetry in young and older adults. Microvascular research 81: 177-182, 2011. 
32. Van Duijnhoven NT, Janssen TW, Green DJ, Minson CT, Hopman MT, Thijssen DH. Effect of functional electrostimulation on impaired skin vasodilator responses to local heating in spinal cord injury. J Appl Physiol (1985) 106: 1065-1071, 2009. 
Table 1. Participant characteristics. $P$-values t-test comparison between males and females. Data are mean $\pm \mathrm{SD}(*=\mathrm{P}<0.05)$

\begin{tabular}{|c|c|c|c|c|}
\hline & $\begin{array}{c}\text { Men } \\
(\mathbf{N}=5)\end{array}$ & $\begin{array}{c}\text { Women } \\
(\mathrm{N}=4)\end{array}$ & $\begin{array}{l}\text { Total } \\
(\mathrm{N}=9)\end{array}$ & P values \\
\hline Age (years) & $26.6 \pm 6.0$ & $27.3 \pm 7.3$ & $26.9 \pm 6.2$ & 0.891 \\
\hline \multicolumn{5}{|l|}{ Ethnicity } \\
\hline - Caucasian & 3 & 4 & 7 & \\
\hline - Asian & 2 & - & 2 & \\
\hline Height (cm) & $174 \pm 4$ & $165 \pm 2$ & $170 \pm 1$ & $0.003^{*}$ \\
\hline Body mass (kg) & $70.0 \pm 5.6$ & $59.3 \pm 5.4$ & $65.2 \pm 7.7$ & $0.025^{*}$ \\
\hline Body mass index $\left(\mathrm{kg} / \mathrm{m}^{2}\right)$ & $23.1 \pm 1.8$ & $21.9 \pm 2.3$ & $22.6 \pm 2.0$ & 0.435 \\
\hline Resting heart rate (beats'min ${ }^{-1}$ ) & $58 \pm 12$ & $65 \pm 6$ & $61 \pm 10$ & 0.286 \\
\hline Mean arterial pressure (mmHg) & $79 \pm 4$ & $82 \pm 10$ & $80 \pm 1$ & 0.670 \\
\hline \multicolumn{5}{|l|}{ Dominant Arm } \\
\hline - $\quad$ Right & 5 & 3 & 8 & \\
\hline - Left & - & 1 & 1 & \\
\hline
\end{tabular}


Table 2: Baseline Skin Perfusion Results.

Resting data presented as absolute and relative flux (in perfusion units PU) and cutaneous vascular conductance (CVC) under different conditions. Data is presented as mean \pm SD. Reproducibility is calculated between-days (Day 1 versus Day 2). Light-grey shading indicates moderate reproducibility (CV: 10-20\%).

\begin{tabular}{lcccc}
\hline & Day1 & Day 2 & $\begin{array}{c}\text { Between-day CV } \\
(\boldsymbol{\%})\end{array}$ & Paired t-test \\
\hline Absolute PU, site 1 & $25 \pm 12$ & $31 \pm 11$ & $23.3(15.9-43.9)$ & $\mathbf{0 . 0 3 7}$ \\
Absolute PU, site 2 & $18 \pm 7$ & $21 \pm 7$ & $21.6(14.8-40.5)$ & 0.146 \\
Relative PU, site 1 & $8 \pm 5$ & $10 \pm 4$ & $28.5(19.4-54.6)$ & 0.133 \\
Relative PU, site 2 & $7 \pm 2$ & $8 \pm 2$ & $19.6(13.4-36.7)$ & 0.087 \\
Absolute CVC, site 1 & $0.31 \pm 0.16$ & $0.40 \pm 0.18$ & $25.5(17.4-48.4)$ & 0.058 \\
Absolute CVC, site 2 & $0.23 \pm 0.09$ & $0.27 \pm 0.11$ & $23.3(15.9-43.8)$ & 0.117 \\
Relative \%CVC max, site 1 & $8 \pm 6$ & $11 \pm 4$ & $30.9(21.0-59.7)$ & 0.074 \\
Relative \%CVCmax, site 2 & $7 \pm 2$ & $8 \pm 2$ & $22.4(15.3-42.0)$ & $\mathbf{0 . 0 3 2}$ \\
\hline \hline
\end{tabular}


Table 3: Local Heating Skin Perfusion Results.

Data presented as absolute and relative flux (in perfusion units (PU)) and cutaneous vascular conductance (CVC) measured during local heating for the 2 different measurements times (Day 1 and Day 2) and during the plateau phases at $42^{\circ} \mathrm{C}$ and $44{ }^{\circ} \mathrm{C}$. Data is presented as means \pm SD Reproducibility is calculated for between-days and presented as CVs ( $\pm 95 \% \mathrm{CI}$ ). Light-grey shading indicates moderate reproducibility (CV: 10-20\%). Dark-grey shading indicates good reporducibility (CV <10\%).

\begin{tabular}{|c|c|c|c|c|}
\hline & Day 1 & Day 2 & $\begin{array}{c}\text { Between-day CV } \\
(\%)\end{array}$ & Paired t-test \\
\hline \multicolumn{5}{|l|}{ Plateau at $42^{\circ} \mathrm{C}$} \\
\hline Absolute PU, site 1 & $271 \pm 57$ & $245 \pm 48$ & $18.1(12.4-33.7)$ & 0.272 \\
\hline Absolute PU, site 2 & $214 \pm 55$ & $208 \pm 67$ & $16.9(11.6-31.3)$ & 0.695 \\
\hline Relative $\% P U_{\max }$, site 1 & $86 \pm 4$ & $82 \pm 10$ & $8.4(5.8-15.4)$ & 0.144 \\
\hline Relative $\% P U_{\max }$, site 2 & $85 \pm 9$ & $81 \pm 13$ & $10.3(7.1-18.9)$ & 0.336 \\
\hline Absolute CVC, site 1 & $3.39 \pm 0.62$ & $3.14 \pm 0.89$ & $18.9(13.0-35.3)$ & 0.394 \\
\hline Absolute CVC, site 2 & $2.71 \pm 0.76$ & $2.67 \pm 1.07$ & $17.1(11.7-31.7)$ & 0.834 \\
\hline Relative $\% C V C_{\max }$, site 1 & $85 \pm 6$ & $83 \pm 11$ & $8.6(5.9-15.8)$ & 0.612 \\
\hline Relative $\% C V C_{\max }$, site 2 & $84 \pm 10$ & $82 \pm 13$ & $9.9(6.8-18.2)$ & 0.698 \\
\hline \multicolumn{5}{|l|}{ Plateau at $44^{\circ} \mathrm{C}$} \\
\hline Absolute PU, site 1 & $314 \pm 63$ & $300 \pm 41$ & $18.1(12.4-33.7)$ & 0.611 \\
\hline Absolute PU, site 2 & $253 \pm 54$ & $255 \pm 56$ & $14.7(10.1-27.1)$ & 0.928 \\
\hline Absolute CVC, site 1 & $3.99 \pm 0.74$ & $3.73 \pm 0.71$ & $16.9(11.6-31.3)$ & 0.412 \\
\hline Absolute CVC, site 2 & $3.25 \pm 0.82$ & $3.18 \pm 0.89$ & $14.4(9.9-26.6)$ & 0.754 \\
\hline
\end{tabular}




\section{Table 4: Co-efficient of variation between sites:}

Data presented are between-site (site 1 versus site 2) reproducibility is calculated during the 2 different measurements times (Day 1 and Day 2). Furthermore, we calculated the between-day CV when using site 1 for the first measurement and site 2 for the second measurement. Data is presented as CVs ( $\pm 95 \%$ CI). Light-grey shading indicates moderate reproducibility (CV: 10-20\%). Dark-grey shading indicates good reporducibility $(\mathrm{CV}<10 \%)$.

\begin{tabular}{|c|c|c|c|}
\hline & $\overline{\text { Day } 1}$ & Day 2 & \\
\hline Baseline $\left(33^{\circ} \mathrm{C}\right)$ & $\begin{array}{c}\mathrm{CV}(\%) \\
\text { site } 1 \text { vs site } 2\end{array}$ & $\begin{array}{c}\text { CV }(\%) \\
\text { site } 1 \text { vs site } 2\end{array}$ & $\begin{array}{c}\text { Between-day CV } \\
(\%) \\
\text { (site } 1 \text { Day1 vs } \\
\text { site } 2 \text { Day } 2 \text { ) }\end{array}$ \\
\hline Absolute flux, $P U$ & $33.8(22.9-65.9)$ & $32.4(22.0-62.8)$ & $30.7(20.9-59.1)$ \\
\hline Relative flux, $P U$ & $29.1(19.8-55.8)$ & $19.8(13.5-36.9)$ & $29.9(20.3-57.4)$ \\
\hline Absolute CVC, AU & $33.8(22.9-65.9)$ & $32.6(22.1-63.3)$ & $30.9(21.0-59.6)$ \\
\hline Relative $\% C V C_{\max }, A U$ & $29.1(19.8-55.8)$ & $20.0(13.7-37.4)$ & $30.9(21.0-59.6)$ \\
\hline \multicolumn{4}{|l|}{ Plateau at $42^{\circ} \mathrm{C}$} \\
\hline Absolute flux, $P U$ & $21.3(14.6-39.9)$ & $20.6(14.1-38.5)$ & $31.6(21.4-61.1)$ \\
\hline Relative flux, $P U$ & $6.3(4.4-11.6)$ & $5.0(3.4-9.1)$ & $11.1(7.6-20.3)$ \\
\hline Absolute CVC, AU & $21.3(14.6-39.9)$ & $20.6(14.1-38.6)$ & $32.2(21.8-62.3)$ \\
\hline Relative $\% C V C_{\max }, A U$ & $6.3(4.4-11.6)$ & $4.8(3.3-8.8)$ & $11.3(7.7-20.8)$ \\
\hline \multicolumn{4}{|l|}{ Plateau at $44^{\circ} \mathrm{C}$} \\
\hline Absolute flux, $P U$ & $20.3(13.9-38.0)$ & $18.5(12.7-34.4)$ & $25.0(17.1-47.4)$ \\
\hline Absolute $C V C, A U$ & $20.3(13.9-38.0)$ & $18.5(12.7-34.4)$ & $25.7(17.5-48.7)$ \\
\hline
\end{tabular}




\section{FIGURE LEGEND}

FIGURE 1. A representative forearm cutaneous flux response during the slow local heating protocol. For our analysis, we have calculated the average flux over the last 5-minutes of: A. baseline period at $33^{\circ} \mathrm{C}, \mathrm{B}$. plateau phase at $42^{\circ} \mathrm{C}$, and C. plateau phase at $44^{\circ} \mathrm{C}$. The first and second arrow indicate the start and finish of the incremental steps involved in the slow heating protocol $\left(0.5^{\circ} \mathrm{C} / 2 \mathrm{~min} 30 \mathrm{~s}\right.$ until $\left.42^{\circ} \mathrm{C}\right)$, respectively. Arrow 2 is followed by 40-minutes for the cutaneous perfusion to reach a stable plateau phase at $42^{\circ} \mathrm{C}$. Arrow three indicates the increment from 42 to $44^{\circ} \mathrm{C}$ to assess maximal perfusion. The two spikes during the baseline and early stage of the slow heating protocol, indicated by the asterisk, are the result of slight movement of the arm.

FIGURE 2. Band-Altman plots of difference between days in CVC against the mean of the measurements in site 1 at $33^{\circ} \mathrm{C}$ site 1 (upper left panel), at $42^{\circ} \mathrm{C}$ (middle left panel) and at $44^{\circ} \mathrm{C}$ (lower left panel). Bland-Altman plots of the difference in CVC\%max against the mean of the measurements in site 1 at $33^{\circ} \mathrm{C}$ (upper right panel) and at $42^{\circ} \mathrm{C}$ (middle right panel). Middle horizontal line denotes mean value and upper and lower lines denote $95 \%$ limits of agreement. Linear regression showed no evidence of proportional bias. 
Figure 1..

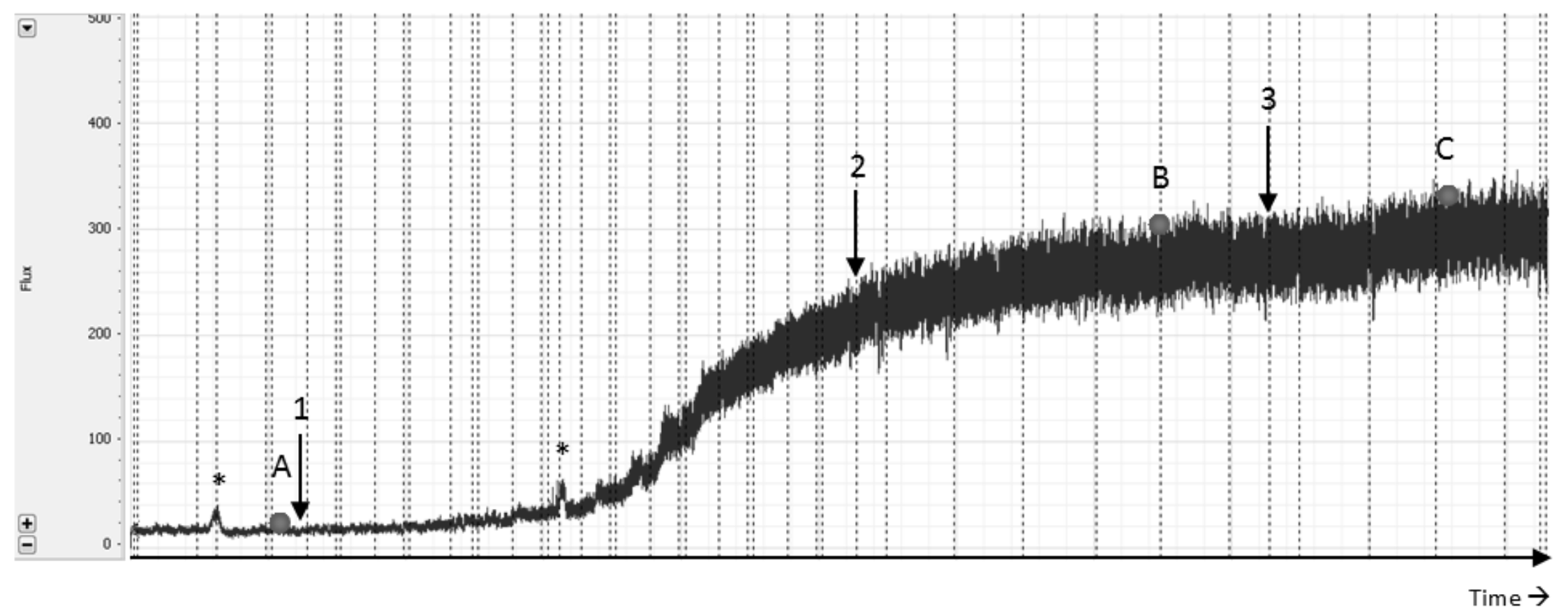


Figure 2
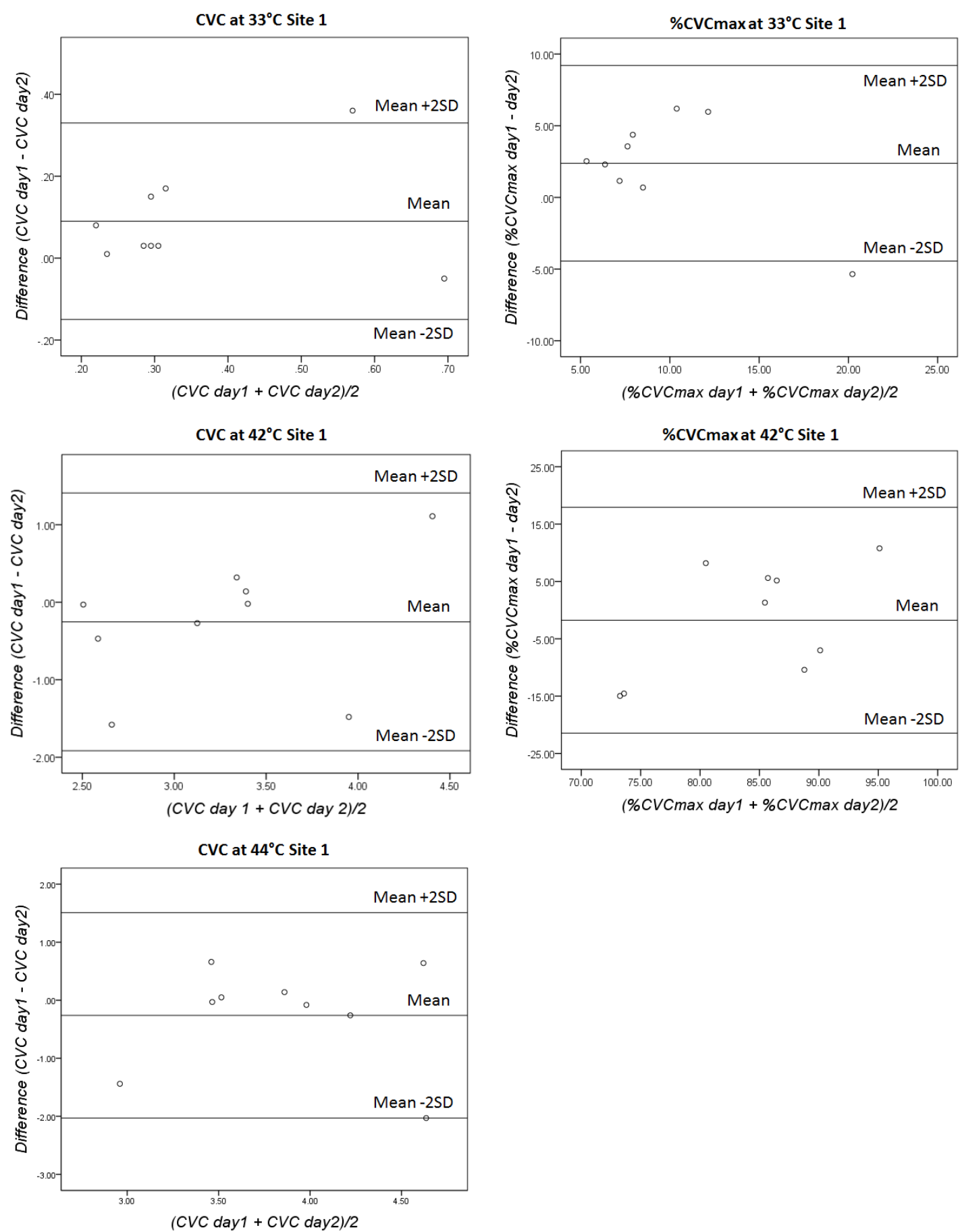